\title{
A randomized controlled trial of Chinese traditional medicine Dachengqi Decoction in the treatment of postoperative intestinal function recovery
}

\author{
Yuwen Luo ${ }^{1,2 \#}$, Xingyu Feng," Deqing $\mathrm{Wu}^{2}$, Junjiang Wang ${ }^{2}$, Zejian Lyv ${ }^{2}$, Jiabin Zheng ${ }^{2}$, \\ Kheng Tian $\mathrm{Lim}^{3}$, Yong $\mathrm{Li}^{1,2}$; on behalf of AME Gastrointestinal Surgery Collaborative Group \\ ${ }^{1}$ Second School of Clinical Medicine, Southern Medical University, Guangzhou, China; ${ }^{2}$ Guangdong Provincial People's Hospital, Guangdong \\ Academy of Medical Sciences, Guangzhou, China; ${ }^{3}$ Department of Surgery, Khoo Teck Puat Hospital, Singapore, Singapore \\ Contributions: (I) Conception and design: Y Li; (II) Administrative support: The Ethics Committee of Guangdong Provincial People's Hospital; (III) \\ Provision of study materials or patients: X Feng, D Wu, J Wang, Z Lyv, J Zheng; (IV) Collection and assembly of data: Y Luo, X Feng; (V) Data \\ analysis and interpretation: Y Luo, X Feng; (VI) Manuscript writing: All authors; (VII) Final approval of manuscript: All authors. \\ "These authors contributed equally to this work. \\ Correspondence to: Yong Li. Department of General Surgery, Guangdong Provincial People's Hospital, Guangdong Academy of Medical Sciences, \\ Guangzhou, China. Email: liyong@gdph.org.cn.
}

Background: Intestinal dysfunction is not conducive to the recovery of patients after surgery. It is particularly important to restore the intestinal function as soon as possible. In recent years, ultrasonic drug penetration therapy as a new type of non-invasive therapy has been used to solve this problem, but its efficacy has not been confirmed.

Methods: Single-centre, parallel, randomized controlled clinical trial in China that included 184 patients undergoing laparoscopic gastrointestinal surgery. Ninety-one participants were randomly assigned to lowfrequency ultrasound and electric pulses for transdermal drug delivery with Dachengqi Decoction (DCQD) (intervention group), and 90 were assigned to the control group after laparoscopic gastrointestinal surgery. The primary outcome was time to first flatus after surgery (by patient's subjective feeling). Secondary outcomes assessed the recovery time of bowel movement, time of the first defecation, postoperative gastrointestinal complications (e.g., nausea, vomit, and bloating), days of hospitalization and treatment costs. Results: Of 184 patients, 181 (98.4\%) completed the trial. The sociodemographic characteristics and efficiency data were comparable in the two groups at baseline. The intervention group had a shorter mean time of bowel movement recovery than the control group [29.4 h (IQR, 22.0-35.0 h) vs. $33.7 \mathrm{~h}$ (IQR, 24.0$40.0 \mathrm{~h} ; \mathrm{P}=0.005)$ ] and a shorter mean time to first flatus after surgery [35.8 h (IQR, 23.1-46.6 h) vs. $46.7 \mathrm{~h}$ (IQR, 25.9-61.3 h; P=0.012)]. Postoperative gastrointestinal reactions (e.g., nausea, vomit, and bloating) occurred in $28.6 \%$ in the intervention group and $43.3 \%$ in the control group $(\mathrm{P}=0.038)$. Two patients in the intervention group had electrical tingling sensations, and one patient had a skin rash during the treatment. There were no significant differences in the occurrence rates of AEs or SAE, days of hospitalization and treatment costs between the two groups.

Conclusions: Low-frequency ultrasound and electric pulses for transdermal drug delivery with DCQD can shorten the time of bowel movement recovery and accelerate first anal exhaust after laparoscopic gastrointestinal surgery.

Trail registration: Chictr.org.cn Identifier: ChiCTR-IPR-17013630.

Keywords: Low-frequency ultrasound; transdermal drug delivery; Dachengqi decoction; intestinal function recovery

Submitted Dec 02, 2019. Accepted for publication Jul 08, 2020.

doi: $10.21037 /$ tcr-19-2671

View this article at: http://dx.doi.org/10.21037/tcr-19-2671 


\section{Introduction}

Postoperative ileus is a significant problem especially after open abdominal surgery via laparotomy and this has reduced since the introduction of laparoscopic surgery. Despite the advancement in laparoscopic surgery, paralytic ileus remains an issue in major complex gastrointestinal surgery due to long operative duration and considerable surgical trauma. The pathogenesis of post-operative ileus involves inhibitory neural reflexes and inflammatory mediators released from the site of injury. The varying degrees of inhibitory function of gastrointestinal motility after surgery may result in the symptoms of abdominal distension, nausea, vomiting, and even intestinal obstruction (1).

The recovery process of gastrointestinal function after surgery mainly includes intestinal paralysis, irregular peristalsis and return to normal motility. It is generally believed that recovery occurs in the first or second day for intestinal paralysis, second to third day for irregular peristalsis, and third to forth day for normal motility (2). The later the recovery of gastrointestinal function, the more severe the pathophysiological changes, such as intestinal effusion, water-electrolyte imbalance, and infection occur. Therefore, it is particularly important to restore the intestinal function of patients after surgery as soon as possible. At present, early oral feeding, ambulation, hot abdominal physiotherapy, anal enema, and Chinese medicine or treatment are commonly used clinically to help patients' intestinal function recovery. In recent years, ultrasonic drug penetration therapy as a new type of noninvasive therapy has been used to solve this problem.

Ultrasound conductance drug penetration therapy (3) is a combination of laser micro-hole technology, ultrasonic transdermal technology, and iontophoresis technology that achieves targeted and fixed-rate precise target drug delivery technology $(4,5)$, which process is simple, safe and noninvasive (6). According to Chinese medicine, DCQD can promote the secretion of Gastrokin, improve disorders of gastric electric rhythm after surgery, reduce the incidence of gastrointestinal motility, and promote the recovery of gastrointestinal motility (7). However, due to the bitter taste of DCQD, oral drinking has limited its clinical application. Therefore, the external form of DCQD as a patch has been developed and combined with the ultrasonic conductivity meter to penetrate the medicine through the abdominal wall; however, this method's efficacy has not been confirmed.

Thus, a parallel randomized controlled clinical trial was designed to evaluate the effect of low frequency ultrasonic transdermal delivery system with DCQD in postoperative intestinal function recovery on patients after laparoscopic gastrointestinal surgery. We present the following article in accordance with the CONSORT reporting checklist (8) (available at http://dx.doi.org/10.21037/tcr-19-2671).

\section{Methods}

\section{Study design and setting}

This single-center and parallel randomized controlled clinical trial took place in the Department of General Surgery of Guangdong Provincial People's Hospital, Guangdong Academy of Medical Sciences in Guangzhou, China. Enrolment occurred between July 1, 2017, and December 31, 2017. This study was approved by the Ethics Committee of Guangdong Provincial People's Hospital (No. GDRE2016429H) and was carried out in adherence with the Declaration of Helsinki (as revised in 2013). All patients involved in the study provided written informed consent.

\section{Participants}

Patients were eligible if they were aged 18-70 years old, underwent elective laparoscopic gastrointestinal surgery, and if the operation time did not exceed six hours. Exclusion criteria included pregnant or lactating women, emergency surgery, extensive metastases of intra-abdominal gastrointestinal tumors or extensive intra-abdominal adhesions, severe abdominal infections, acute complete intestinal obstruction, required colostomy or small intestine ostomy that did not allow for evaluation of bowel exhaust or defecation, severe cardiovascular and haematopoietic diseases, diabetes with poor glycaemic control (fasting blood glucose $>8 \mathrm{mmol} / \mathrm{L}$ ), alanine aminotransferase (ALT) $>$ normal upper limit 1.5 times or creatinine $(\mathrm{CREA})>$ normal upper limit, body mass index (BMI $>30$ or $<15$ ), pacemakers, artificial stents and artificial valves, severe complications or serious infections or unconsciousness on the first postoperative day, use of drugs that affected gastrointestinal motility within one week before surgery, and inclusion in another trial within 4 weeks prior to enrolment.

\section{Sample size estimation and randomization}

Sample size estimation was performed according to the superiority trial design by PASS 11 software. According to the literature results, the postoperative flatus time was $53.6 \pm 10.7$ and $62.9 \pm 11.4 \mathrm{~h}$ in the intervention group and 
the blank control group, respectively. We set the equal ratio $\mathrm{k}=1$, the superiority boundary value $\Delta$ as four hours, type I error $\alpha=0.025$ and type II error $\beta=0.20$; thus, it could be concluded that there were 60 patients in each group. With an expulsion rate of $20 \%$ and a specific expected rejection rate of $6 \%, 92$ patients were enrolled in one group for the study. The data manager used SAS (Statistical Analysis System) to create the randomization sequence with a 1:1 allocation ratio. Two professional physician assistants were arranged to implement patient enrolment, treatment, data collection, and recording. Surgeons who performed the surgery were unaware of treatment allocation throughout the trial.

\section{Interventions}

Briefly, patients in the intervention group received lowfrequency ultrasound and electric pulses for transdermal drug delivery with DCQD treatment after the operation. DCQD is composed of Da Huang (Radix et Rhizoma Rhei), Houpu (Cortex Magnoliae Officinalis), Zhi Shi (Fructus Aurantii Immaturus) and Mang Xiao (Natrii Sulphas). Materials and drugs were provided by "Beijing Noah Tongzhou Medical Technology Co, Ltd." and included the NAVA-01TD ultrasound conductivity meter (Figure S1), ultrasound conductance gel patches, and DCQD patches, as shown in (Figure S2). Patients in the intervention group received the treatment within six hours after the operation, and the DCQD patch was placed on the left abdomen of the patients (Figure S3). Each treatment lasted for 30 minutes, and the DCQD patch was kept in the patient's abdomen for two hours. The procedure was performed once on the day of surgery and twice daily after the first day after surgery until the patient complained of first flatus. The control group received similar conventional treatment that excluded low-frequency ultrasound and electric pulses for transdermal drug delivery with DCQD treatment.

\section{Outcomes}

The primary outcome was defined as the time to first flatus by the patient's subjective feeling after surgery. The secondary outcomes assessed the recovery time of bowel movement (by auscultation of bowel sounds once every 4 hours; if two consecutive results $\geq 4$ times per minute; next, the bowel movement was determined to be recovered), time of the first defecation, postoperative gastrointestinal complications (e.g., nausea, vomit, and bloating), days of hospitalization and treatment costs. We evaluated vital signs, such as body temperature, pulse, and arterial blood pressure, and serum tests, including ALT, AST, CREA, and CRP, before surgery and at days one, three and five after surgery. Adverse events (AEs) and serious adverse events (SAE) were recorded in the Case Report Form (CRF).

\section{Statistical analysis}

All statistical tests used two-sided tests (except for special instructions). Quantitative data was statistically described using case number, mean, standard deviation, median, upper and lower quartiles, and $95 \%$ confidence intervals. The $t$-test was used to compare the difference between the mean measurements of the standard measurement data and the rank sum test. Qualitative data was statistically described using various types of cases and percentages. A comparative analysis of the two groups before and after treatment within the groups or between the groups used the $\chi^{2}$ test, Fisher's exact test or rank sum test. The efficiency of measured data was analyzed based on a full analysis set (FAS) and per protocol set (PPS), while safety measures were examined in the safety set (SS). According to the unilateral, there was a $97.5 \%$ confidence interval upper limit and the superiority boundary $(\Delta)$ between the two groups, whether the efficacy of the intervention group was better than that of the control group was evaluated. If the upper limit of the $97.5 \%$ confidence interval of the two groups was $<-\Delta$, then the effect of the intervention group was better than that of the control group. If the upper limit of the $97.5 \%$ confidence interval of the two groups was $\geq-\Delta$, the curative effect of the intervention group could not be indicated.

\section{Results}

\section{Study population}

During the study period, 351 patients were screened for eligibility and 184 were randomized (Figure 1). Subsequent exclusion of the 3 randomized patients due to laparotomy, great omentum resection only, or small intestine ostomy left 91 patients in the intervention group and 90 in the control group. Table 1 compares the patients' characteristics between the groups, which were well-balanced.

\section{Outcomes assessment}

\section{Primary outcomes}

Postoperative time to first flatus differed significantly 


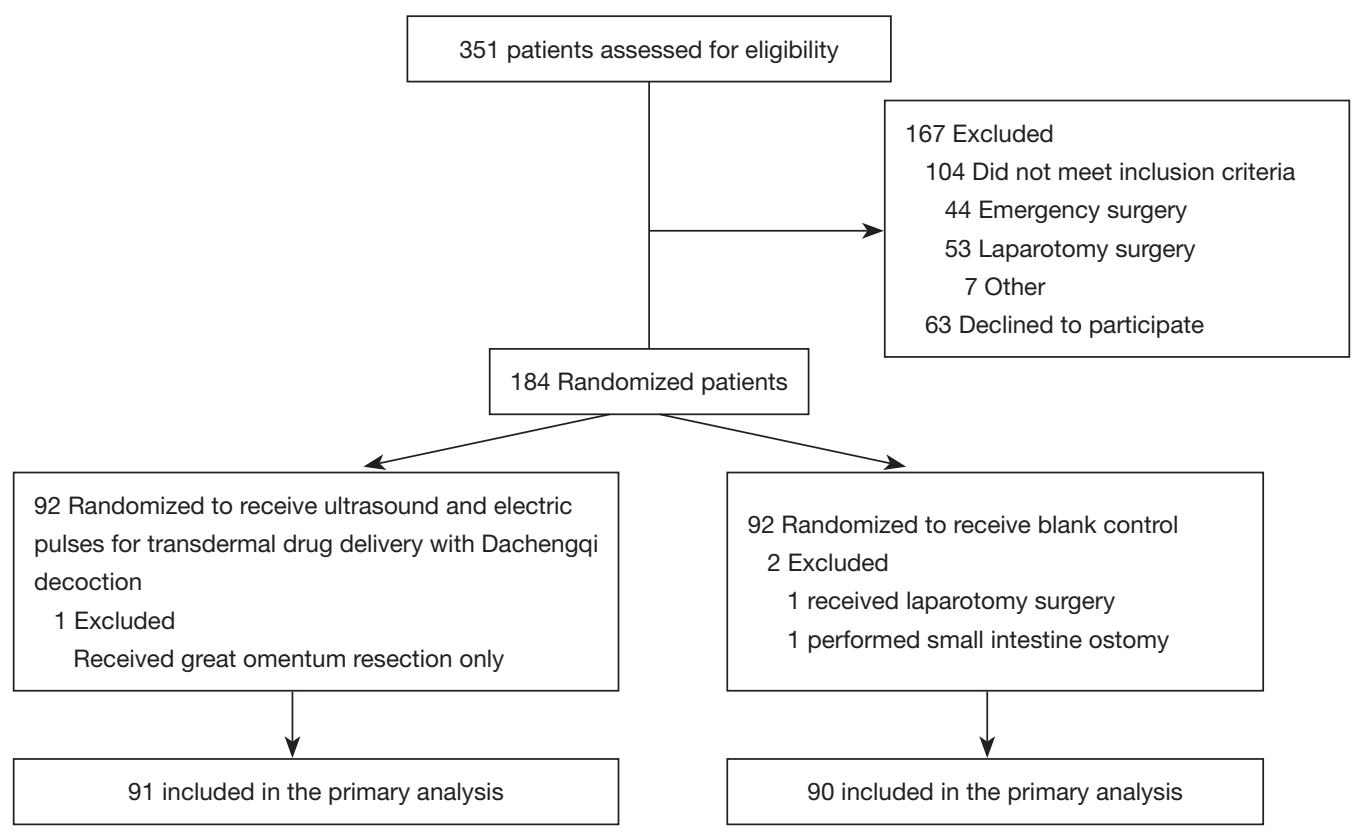

Figure 1 Population flowchart of intervention treatment to laparoscopic gastrointestinal surgery patients.

Table 1 Baseline characteristics

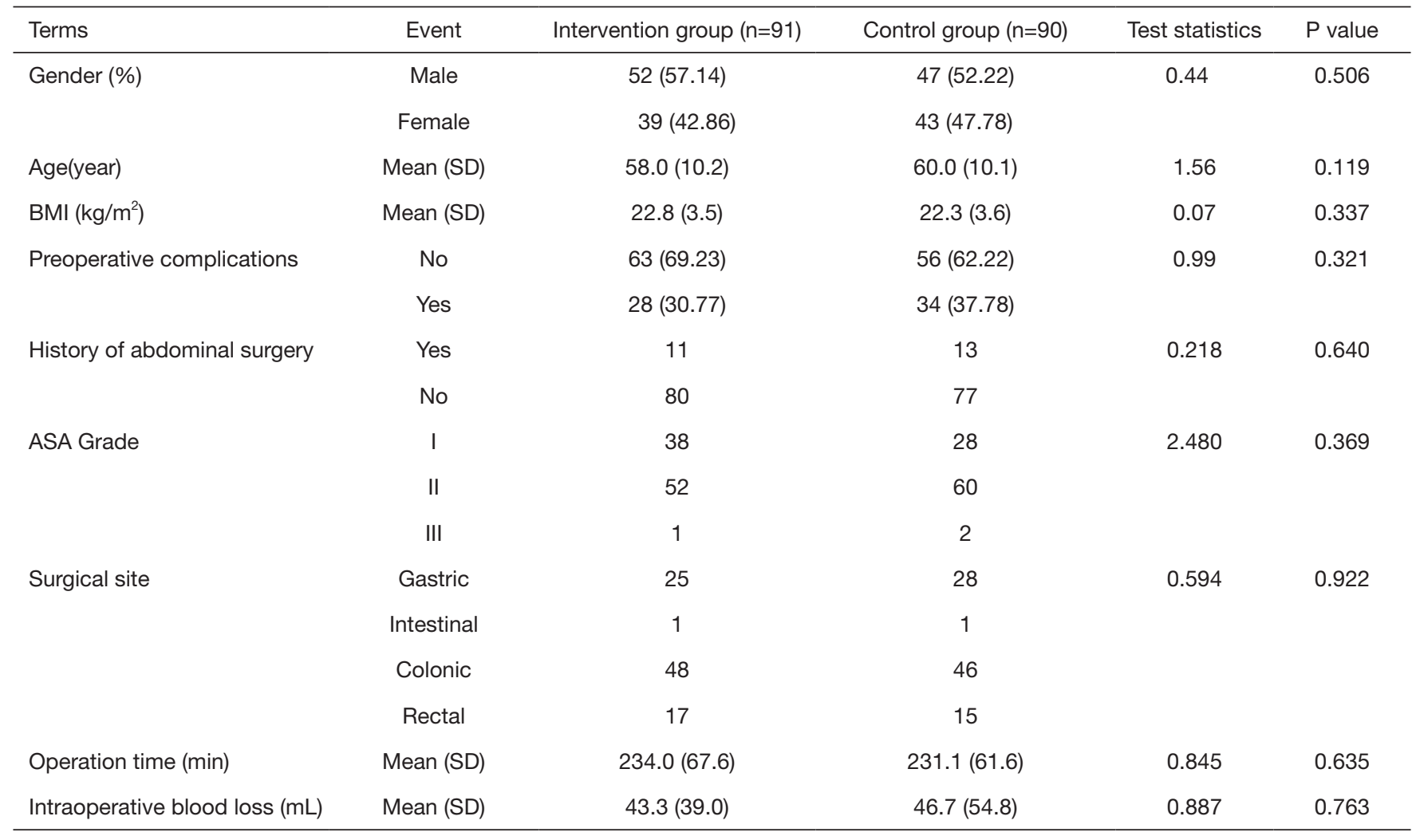


Table 2 Primary outcome and secondary exploratory outcomes

\begin{tabular}{|c|c|c|c|c|c|}
\hline Index & Classification & Group intervention $(n=91)$ & Group control $(n=90)$ & Test ${ }^{\#}$ statistics & $P$ value \\
\hline First defecation time after operation (day) & Mean (SD) & $3.3(1.6)$ & $3.3(1.8)$ & $-0.15^{\mathrm{a}}$ & 0.880 \\
\hline Time of bowel movement recovery (hour) & Mean (SD) & $29.4(11.3)$ & $33.7(12.8)$ & $2.80^{\mathrm{a}}$ & 0.005 \\
\hline Intestinal obstruction & No & $89(97.8)$ & $86(95.6)$ & $--^{\mathrm{b}}$ & 0.444 \\
\hline Number of intravenous infusion days (day) & Mean (SD) & $5.6(2.7)$ & $5.9(3.0)$ & 0.69 & 0.488 \\
\hline Postoperative hospital stayed (day) & Mean (SD) & $6.4(2.8)$ & $6.9(3.1)$ & 0.87 & 0.385 \\
\hline Hospitalization costs (Ten thousand RMB) & Mean (SD) & $6.68(2.27)$ & $6.81(1.71)$ & 0.54 & 0.590 \\
\hline
\end{tabular}

Table 3 Test of optimality for the main effective index (FAS/PPS population)

\begin{tabular}{ccccc}
\hline Time of first anal exhaust (hour) & & Boundary value (h) & \multicolumn{2}{c}{$97.5 \%$ confidence interval for the mean difference (\%) } \\
\cline { 1 - 1 } Group intervention & Group control & & Lower limit \\
35.82 & 46.67 & -4 & -17.62 \\
\hline
\end{tabular}

Table 4 Comparison of postoperative gastrointestinal reactions in two groups

\begin{tabular}{|c|c|c|c|c|c|}
\hline Index & Group intervention $(n=91)$ & Group control $(\mathrm{n}=90)$ & Total $(n=181)$ & Test statistics & $P$ value \\
\hline Yes (\%) & 26 (28.6) & 39 (43.3) & 65 (35.9) & & \\
\hline
\end{tabular}

between the two groups: $35.8 \pm 17.6 \mathrm{~h}$ in the intervention group vs. $46.7 \pm 23.7 \mathrm{~h}$ in the control group, $\mathrm{P}=0.012$ (Table 2). Regarding the superiority test for the main effective indicator, the $97.5 \%$ confidence interval for the mean difference was $-4.0(-17.26,-4.07)$, indicating that the intervention group was superior to control group, which was consistent with the PPS (Per-protocol set) results (Table 3).

\section{Secondary outcomes}

In the intervention group, the time of bowel movement recovery was $29.4 \mathrm{~h}$ (IQR, 22.0-35.0 h), which was significantly shorter than that of the control group (33.7 h, IQR, 24.0-40.0 h; P=0.005) (Table 2). Postoperative gastrointestinal reactions (e.g., nausea, vomit, and bloating) occurred in $28.6 \%$ in the intervention group and $43.3 \%$ in of the control group $(\mathrm{P}=0.038)$, which was determined to be an insignificant difference (Table 4).
The first postoperative defecation time was 3.3 days (IQR, 2.0 4.0 days) in the intervention group vs. 3.3 days (IQR, 2.0-4.0 days) for the control group $(\mathrm{P}=0.880)$. The incidence of intestinal obstruction was $2.2 \%$ in the intervention group and $4.4 \%$ in the control group ( $\mathrm{P}=0.444)$. The impact of postoperative complications in the intervention group was $15.4 \%$ and $23.3 \%$ in the control group $(\mathrm{P}=0.176)$, which was determined to be an insignificant difference. Days of hospitalization and treatment costs were similar between the two groups (Table 2).

Changes from baseline over time in alanine aminotransferase, aspartate aminotransferase, C-reactive protein, and Creatinine within each group are illustrated in Figure 2, which demonstrated no significant difference. Vital signs (Body temperature, Heart rate, Systolic pressure, and Diastolic pressure) results were summarized in Table S1, and Serological examinations (WBC, HGB and PLT) were summarized in Table S2, which showed no significant 
A

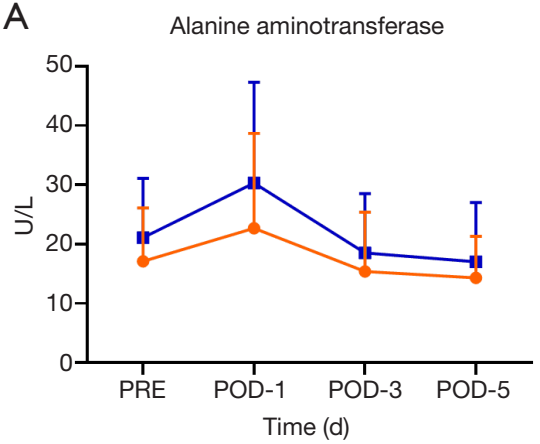

C

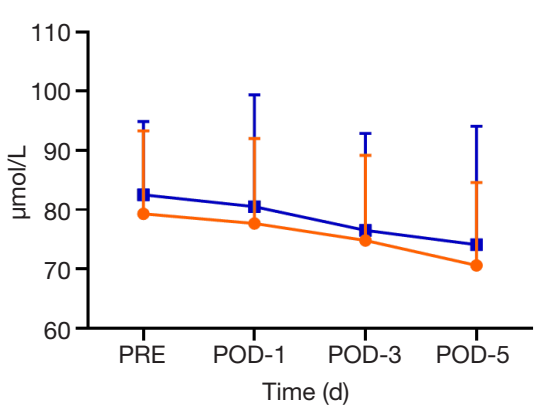

$$
\begin{aligned}
& \text { - Intervention group } \\
& \text { - Control group }
\end{aligned}
$$

B Aspartate aminotransferase

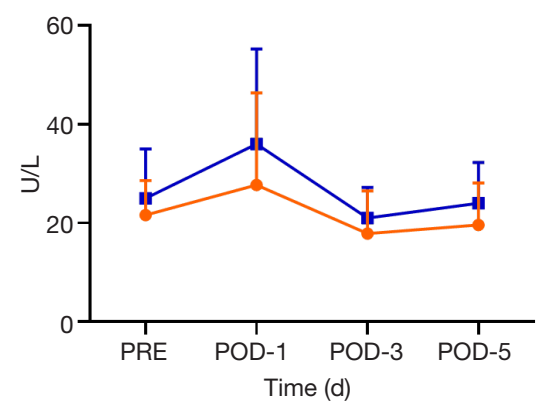

D

$\rightarrow-$ Intervention group

- Control group

Figure 2 Changes in serum test indices from baseline over time. PRE, preoperative; POD-1, postoperative day first; POD-3, postoperative day third; POD-5, postoperative day fifth.

difference in the two groups.

\section{Adverse events}

Adverse events were reported for 26 of 91 patients (28.6\%) and 39 of 90 patients $(43.3 \%)$, while severe adverse events were reported for 11 of 91 patients $(12.1 \%)$ and 14 of 90 patients $(15.6 \%)$ in the intervention group and control group, respectively (Table 5). No patients died during the perioperative period, which was defined as extending from hospitalization to discharge for one week.

\section{Discussion}

To the best of our knowledge, this is the first randomized controlled clinical trial to evaluate low-frequency ultrasound and electric pulses for transdermal drug delivery with DCQD treatment for patients after a laparoscopic gastrointestinal surgery. This design reflects the daily practice of the combination of Chinese and Western medicine treatment options in many centers of China (9-12).
This trial found significant differences in the time of the recovery of bowel movement and time to first flatus after laparoscopic gastrointestinal surgery between the group with low-frequency ultrasound and electric pulses for transdermal drug delivery with DCQD and the control group. Previous studies suggested that DCQD can increase plasma motilin, enhance gastrointestinal motility, improve gastric dysrhythmia, and reduce gastroparesis after an abdominal surgery (13-15). In the current study, we did not routinely test the plasma motilin, but we found that postoperative time to first flatus did shorten about $10.9 \mathrm{~h}$. In the Pan (16) review, it was found that DCQD can reduce capillary endothelial damage in acute pancreatitis-associated intestinal injury and the mechanism may be associated with the regulation of endothelial barrier function-associated proteins AQP-1, MMP9, and JAM-C. Thus, we speculated that the DCQD could accelerate the recovery of intestinal function by reducing inflammation $(5,17,18)$.

Although the results showed no significant difference in terms of postoperative intestinal obstruction (POI) (19), the risk of adverse events associated with postoperative 
Table 5 Adverse events and severe adverse events

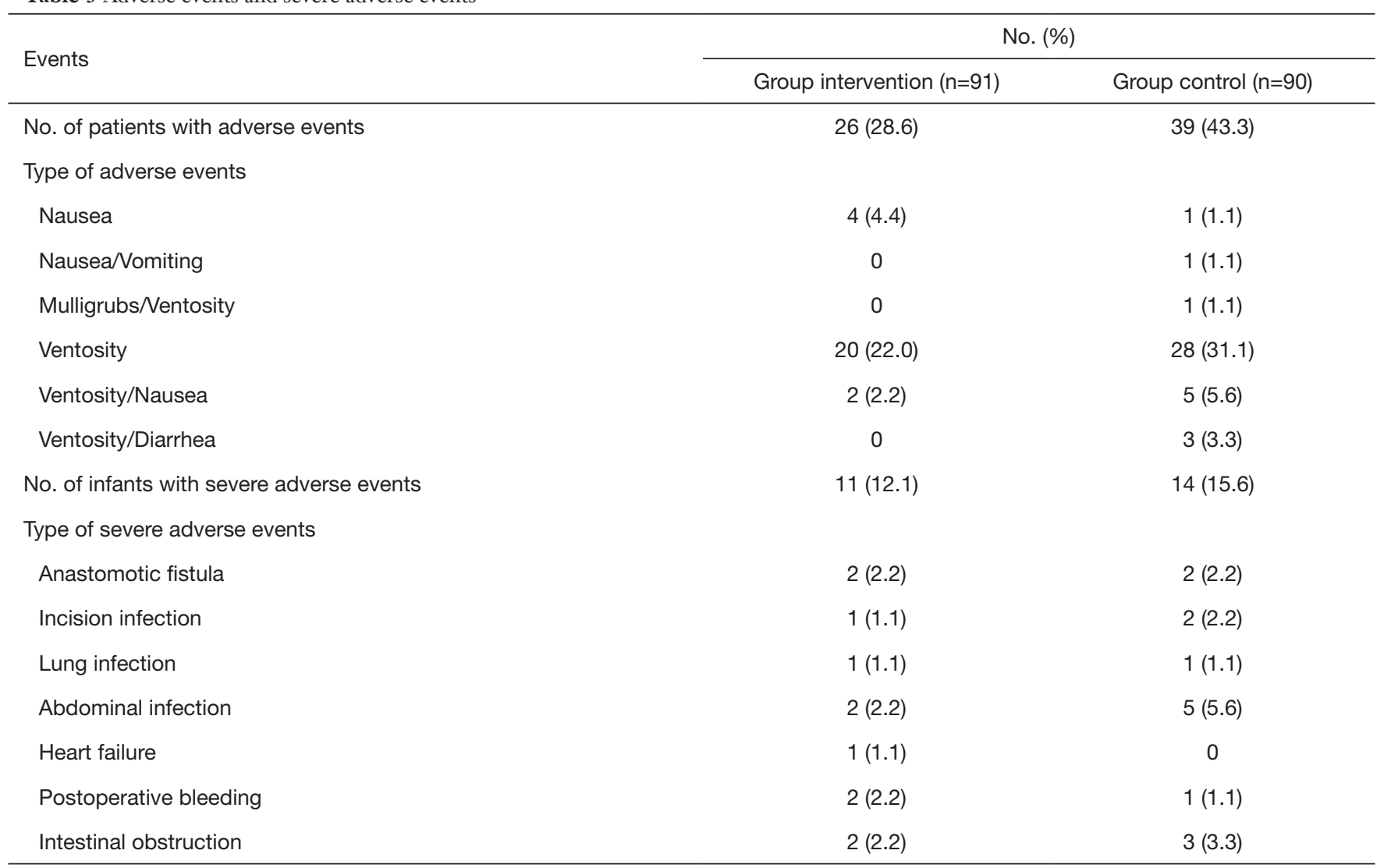

gastrointestinal reactions (nausea, vomit, bloating, and others) occurred in $28.6 \%$ of the tested patients in the intervention group and $43.3 \%$ in the control group $(\mathrm{P}=0.038)$, which was shown to be an insignificant difference. Meta-analysis involved 664 participants from five randomized trials suggesting that patients receiving a traditional Chinese medication method combined with a conventional therapy seemed to have improved outcomes compared with the patients receiving a standard treatment alone (OR 4.24; 95\% CI, 2.83 to 6.36) (20), which still lacks the support of advantageous evidence.

Safety needs to be considered when implementing a treatment. The type of severe adverse events did not show a significant difference between the two groups, and it was usually thought to be associated with surgical quality $(21,22)$. During the treatment course, there were three adverse events $(3.3 \%)$ caused by intervention, where two patients had electrical tingling sensation during the treatment. After adjusting the frequency of treatment, they were able to tolerate the follow-up treatment. In 1 case, there was a skin rash due to the affixed electrode during surgery, which was also observed in areas where the electrocardiographic monitoring electrodes were placed. The reactions were considered as an allergy to the medical tape. The rest of the patients had no pruritus, swelling, ulceration, or other adverse reactions. In this study, we routinely detected changes from baseline over time in liver and kidney function before and after surgery. There was no significant difference between the two groups $(\mathrm{P}>0.05)$ concerning ALT, AST, CREA, and CRP. Studies showed that the DCQD could reduce the degree of liver and kidney function damage in rats $(23,24)$. Therefore, we believe that low-frequency ultrasound conduction combined with DCQD would not affect the function of the liver and kidney.

In summary, low-frequency ultrasound and electric pulses for transdermal drug delivery with traditional Chinese medicine DCQD can improve abdominal distention symptoms in patients after laparoscopic gastrointestinal surgery and shorten the recovery time of the gastrointestinal function. The implementation process was simple, and the degree of patient cooperation was high; thus, it can be a strategy to enhance patient recovery after surgery. 


\section{Limitations}

This study has several limitations. First, the trial was a singlecenter, randomized controlled clinical trial and not doubleblinded, and thus there was a certain degree of bias. Further comprehensive verification and evidence from multi-center clinical studies are required. Second, it may be beneficial to have a placebo group in this study, where the low frequency ultrasonic transdermal delivery system with DCQD patch should be performed on a control group of patients without the treatment power on. Third, this study was a manifestation of the combination of Chinese and Western medicine treatment, and so although we achieved expected results in laparoscopic surgery patients, further studies are needed to prove the efficacy for laparotomy patients. Fourth, there were some heterogeneous types of surgical resection (gastric versus colorectal) in both arms of study, of which we have closely matched to reduce the bias. (Worth describing whether these were total/subtotal gastrectomy or right/left/ subtotal colectomy, anterior resections etc. in Table 1).

\section{Conclusions}

The result of this study supported that low-frequency ultrasound and electric pulses for transdermal drug delivery with the DCQD can shorten the time of bowel movement recovery and accelerate time to first flatus after a laparoscopic gastrointestinal surgery. Furthermore, it is safe in clinical usage and has prospective clinical applications.

\section{Acknowledgments}

Beijing Noah Tongzhou Medical Technology Co, Ltd. supported the medications and apparatus, but played no role in the top-level design, clinical practice, data collection, trial supervision, and data analysis.

Funding: The trail was supported by the Research Fund of Guangdong Provincial People's Hospital (No. KY012019130).

\section{Footnote}

Reporting Checklist: The authors have completed the CONSORT reporting checklist. Available at http://dx.doi. org/10.21037/tcr-19-2671

Data Sharing Statement: Available at http://dx.doi. org/10.21037/tcr-19-2671
Conflicts of Interest: All authors have completed the ICMJE uniform disclosure form (available at http://dx.doi. org/10.21037/tcr-19-2671). XF reports grants from the Research Fund of Guangdong Provincial People's Hospital (No. KY012019130), outside the submitted work. The other authors have no conflicts of interest to declare.

Ethical Statement: The authors are accountable for all aspects of the work in ensuring that questions related to the accuracy or integrity of any part of the work are appropriately investigated and resolved. The study was conducted in accordance with the Declaration of Helsinki (as revised in 2013). The study was approved by the Ethics Committee of Guangdong Provincial People's Hospital (No. GDRE2016429H) and informed consent was taken from all the patients.

Open Access Statement: This is an Open Access article distributed in accordance with the Creative Commons Attribution-NonCommercial-NoDerivs 4.0 International License (CC BY-NC-ND 4.0), which permits the noncommercial replication and distribution of the article with the strict proviso that no changes or edits are made and the original work is properly cited (including links to both the formal publication through the relevant DOI and the license). See: https://creativecommons.org/licenses/by-nc$\mathrm{nd} / 4.0 /$.

\section{References}

1. Kim MC, Kim W, Kim HH, et al. Risk factors associated with complication following laparoscopy-assisted gastrectomy for gastric cancer: a large-scale korean multicenter study. Ann Surg Oncol 2008;15:2692-700.

2. Fruhwald S, Herk E, Hammer HF, et al. Differential reversal of drug-induced small bowel paralysis by cerulein and neostigmine. Intensive Care Med 2004;30:1414-20.

3. Klibanov AL, Hossack JA. Ultrasound in Radiology: From Anatomic, Functional, Molecular Imaging to Drug Delivery and Image-Guided Therapy. Invest Radiol 2015;50:657-70.

4. Peng HM, Zhu PC, Chen ZJ. Thermal analyses of in vitro low frequency sonophoresis. Ultrason Sonochem 2017;35:458-70.

5. Zhang YM, Zhu L, Zhao XL, et al. Optimal timing for the oral administration of Da-Cheng-Qi decoction based on the pharmacokinetic and pharmacodynamic targeting of the pancreas in rats with acute pancreatitis. World J 
Gastroenterol 2017;23:7098-109.

6. Boonhong J, Suntornpiyapan P, Piriyajarukul A. Ultrasound combined transcutaneous electrical nerve stimulation (UltraTENS) versus phonophoresis of piroxicam $(\mathrm{PhP})$ in symptomatic knee osteoarthritis: A randomized double-blind, controlled trial. J Back Musculoskelet Rehabil 2018;31:507-13.

7. Pan LY, Chen YF, Li HC, et al. Dachengqi Decoction Attenuates Intestinal Vascular Endothelial Injury in Severe Acute Pancreatitis in Vitro and in Vivo. Cell Physiol Biochem 2017;44:2395-406.

8. Schulz KF, Altman DG, Moher D, et al. CONSORT 2010 statement: updated guidelines for reporting parallel group randomised trials. Int J Surg 2011;9:672-7.

9. Cai QH, Ma R, Hu SY, et al. A Randomized Controlled Trial of Chinese Patent Medicine Xiao'er Biantong Granules in the Treatment of Functional Constipation in Children. Evid Based Complement Alternat Med 2018;2018:4941505.

10. Jiang DL, Yang J, Jiang SY, et al. Modified Da Chengqi granules improvement in immune function in early severe acute pancreatitis patients. Genet Mol Res 2016;15:10.4238/gmr.15028787.

11. Yang G, Qiu J, Wang D, et al. Traditional Chinese Medicine Curcumin Sensitizes Human Colon Cancer to Radiation by Altering the Expression of DNA Repairrelated Genes. Anticancer Res 2018;38:131-6.

12. Leng $Y$, Gao H, Fu X, et al. The efficacy and safety of Chinese herbal medicine Shen-Qi Hua-Yu formula in patients with diabetic lower extremity artery disease: Study protocol of a multi-center, randomized, doubleblind, placebo-controlled trial. Medicine (Baltimore) 2020;99:e18713.

13. Qi QH, Wang J, Liang GG, et al. Da-Cheng-Qi-Tang promotes the recovery of gastrointestinal motility after abdominal surgery in humans. Dig Dis Sci 2007;52:1562-70.

14. Jian W, Heng L, Hui QQ. Effect of Da-Cheng-QiTang on gastrointestinal motility in patients undergoing laparotomy. Hepatogastroenterology 2011;58:1887-92.

15. Luo P, Xie M, Qi Q. Mitigation of the damage of intestinal smooth muscle of rats with multiple organ dysfunction syndrome by Dachengqi decoction through mitochondrial pathway. Zhonghua Wei Zhong Bing Ji Jiu Yi Xue 2015;27:106-9.

16. Pan LY, Chen YF, Li HC, et al. Dachengqi Decoction Attenuates Intestinal Vascular Endothelial Injury in Severe
Acute Pancreatitis in Vitro and in Vivo. Cell Physiol Biochem 2017;44:2395-406.

17. Chen Z, Chen Y, Pan L, et al. Dachengqi Decoction Attenuates Inflammatory Response via Inhibiting HMGB1

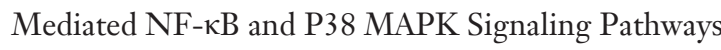
in Severe Acute Pancreatitis. Cell Physiol Biochem 2015;37:1379-89.

18. Hu X, Liu S, Zhu J, et al. Dachengqi decoction alleviates acute lung injury and inhibits inflammatory cytokines production through TLR4/NF- $\kappa \mathrm{B}$ signaling pathway in vivo and in vitro. J Cell Biochem 2019;120:8956-64.

19. Nakamura T, Sato T, Naito M, et al. Laparoscopic Surgery is Useful for Preventing Recurrence of Small Bowel Obstruction After Surgery for Postoperative Small Bowel Obstruction. Surg Laparosc Endosc Percutan Tech 2016;26:e1-4.

20. Suo T, Gu X, Andersson R, et al. Oral traditional Chinese medication for adhesive small bowel obstruction. Cochrane Database Syst Rev 2012;(5):CD008836.

21. Li YW, Lian P, Huang B, et al. Very Early Colorectal Anastomotic Leakage within 5 Post-operative Days: a More Severe Subtype Needs Relaparatomy. Sci Rep 2017;7:39936.

22. Yu J, Huang C, Sun Y, et al. Effect of Laparoscopic vs Open Distal Gastrectomy on 3-Year Disease-Free Survival in Patients With Locally Advanced Gastric Cancer: The CLASS-01 Randomized Clinical Trial. JAMA 2019;321:1983-92.

23. Luo P, Xie M, Qi Q. Mitigation of the damage of intestinal smooth muscle of rats with multiple organ dysfunction syndrome by Dachengqi decoction through mitochondrial pathway. Zhonghua Wei Zhong Bing Ji Jiu Yi Xue 2015;27:106-9.

24. Wang FL, Yang HZ, Li YM, et al. Prevention and treatment mechanism of qingxia therapy (based on yinchenhao decoction and dachengqi decoction) on hepatocyte apoptosis in rats with acute hepatic injury induced by lipopolysaccharide/D-galactosamine. Zhong Yao Cai 2014;37:848-52.

Cite this article as: Luo Y, Feng X, Wu D, Wang J, Lyv Z, Zheng J, Lim KT, Li Y; on behalf of AME Gastrointestinal Surgery Collaborative Group. A randomized controlled trial of Chinese traditional medicine Dachengqi Decoction in the treatment of postoperative intestinal function recovery. Transl Cancer Res 2020;9(8):4498-4506. doi: 10.21037/tcr-19-2671 


\section{Supplementary}

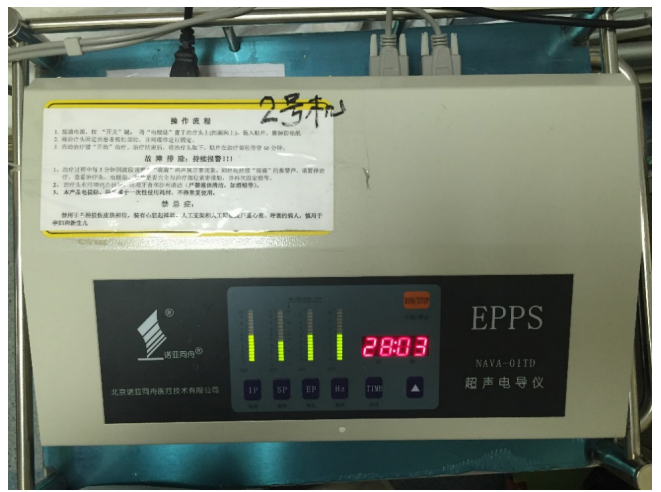

Figure S1 The NAVA-01TD ultrasound and electric pulses for transdermal drug delivery.

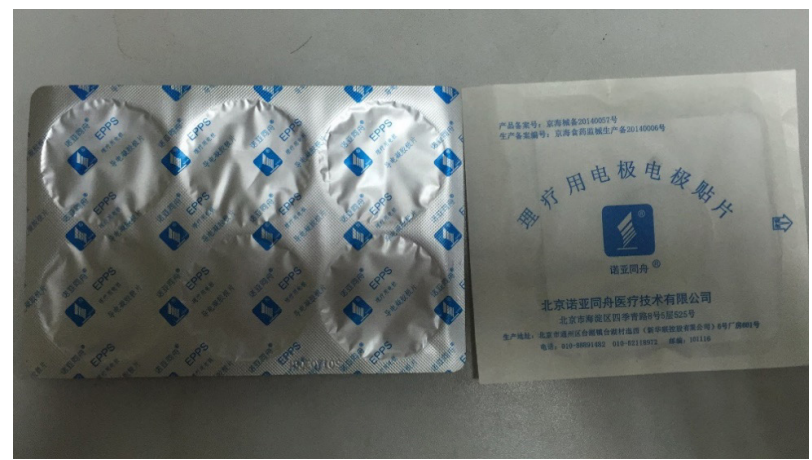

Figure S2 The Dachengqi Decoction patch.

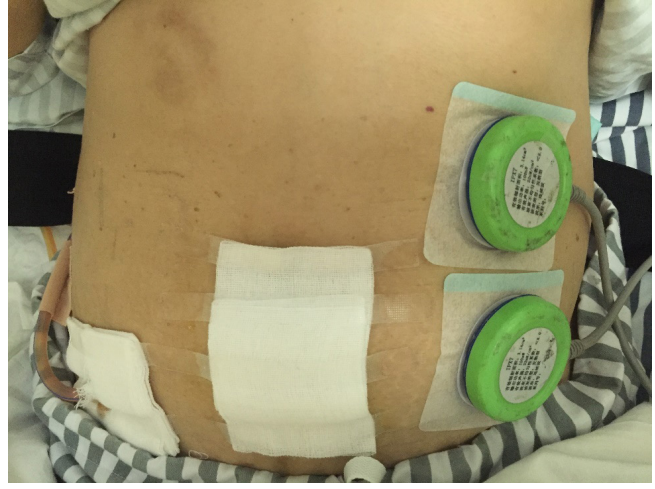

Figure S3 The Dachengqi Decoction patch paste position schematic. 


\begin{tabular}{|c|c|c|c|c|c|}
\hline Index & Classification & Statistics & Group intervention, 91 & Group control, 90 & Total \\
\hline \multirow{15}{*}{$\begin{array}{l}\text { Body } \\
\text { temperature } \\
\left({ }^{\circ} \mathrm{C}\right)\end{array}$} & \multirow[t]{3}{*}{ Pre-operation } & Mean (SD) & $36.61(0.26)$ & $36.65(0.25)$ & $36.63(0.25)$ \\
\hline & & Median (Q1, Q3) & $36.60(36.50,36.80)$ & $36.60(36.50,36.80)$ & $36.60(36.50,36.80)$ \\
\hline & & Min, Max & $36.10,37.30$ & $36.20,37.40$ & $36.10,37.40$ \\
\hline & \multirow[t]{3}{*}{ POD-1 } & Mean (SD) & $36.93(0.40)$ & $36.91(0.36)$ & $36.92(0.38)$ \\
\hline & & Median (Q1, Q3) & $36.90(36.70,37.20)$ & $36.80(36.70,37.10)$ & $36.90(36.70,37.10)$ \\
\hline & & Min, Max & $35.30,38.40$ & $36.10,38.00$ & $35.30,38.40$ \\
\hline & \multirow[t]{3}{*}{ POD-2 } & Mean (SD) & $36.80(0.34)$ & $36.84(0.33)$ & $36.82(0.34)$ \\
\hline & & Median (Q1, Q3) & $36.80(36.50,37.00)$ & $36.80(36.60,37.00)$ & $36.80(36.60,37.00)$ \\
\hline & & Min, Max & $36.10,38.40$ & $36.10,38.00$ & $36.10,38.40$ \\
\hline & \multirow[t]{3}{*}{ POD-3 } & Mean (SD) & $36.73(0.28)$ & $36.72(0.27)$ & $36.73(0.28)$ \\
\hline & & Median (Q1, Q3) & $36.70(36.50,36.90)$ & $36.70(36.50,36.90)$ & $36.70(36.50,36.90)$ \\
\hline & & Min, Max & $36.10,37.80$ & $36.00,37.40$ & $36.00,37.80$ \\
\hline & \multirow[t]{3}{*}{ POD-4 } & Mean (SD) & $36.55(0.35)$ & $36.62(0.30)$ & $36.58(0.33)$ \\
\hline & & Median (Q1, Q3) & $36.50(36.30,36.80)$ & $36.60(36.50,36.80)$ & $36.60(36.40,36.80)$ \\
\hline & & Min, Max & $36.00,37.80$ & $36.00,38.00$ & $36.00,38.00$ \\
\hline \multirow{15}{*}{$\begin{array}{l}\text { Heart rate } \\
\text { (times/ } \\
\text { minute) }\end{array}$} & \multirow[t]{3}{*}{ Pre-operation } & Mean (SD) & $79.11(11.39)$ & $80.37(9.71)$ & $79.73(10.58)$ \\
\hline & & Median (Q1, Q3) & $78.00(72.00,86.00)$ & $79.50(74.00,88.00)$ & $78.00(72.00,87.00)$ \\
\hline & & Min, Max & $52.00,113.00$ & $60.00,109.00$ & $52.00,113.00$ \\
\hline & \multirow[t]{3}{*}{ POD-1 } & Mean (SD) & $83.24(12.34)$ & $81.21(10.11)$ & $82.23(11.30)$ \\
\hline & & Median (Q1, Q3) & $82.00(76.00,89.00)$ & $80.00(75.00,88.00)$ & $81.00(75.00,88.00)$ \\
\hline & & Min, Max & $64.00,140.00$ & $62.00,108.00$ & $62.00,140.00$ \\
\hline & \multirow[t]{3}{*}{ POD-2 } & Mean (SD) & $84.58(13.14)$ & $81.82(12.20)$ & $83.20(12.72)$ \\
\hline & & Median (Q1, Q3) & $82.00(76.00,92.00)$ & $80.00(76.00,88.00)$ & $80.00(76.00,90.00)$ \\
\hline & & Min, Max & $61.00,128.00$ & $38.00,122.00$ & $38.00,128.00$ \\
\hline & \multirow[t]{3}{*}{ POD-3 } & Mean (SD) & $82.87(12.40)$ & $81.93(11.66)$ & $82.40(12.01)$ \\
\hline & & Median (Q1, Q3) & $82.00(76.00,88.00)$ & $80.00(76.00,88.00)$ & $80.00(76.00,88.00)$ \\
\hline & & Min, Max & $55.00,124.00$ & $60.00,130.00$ & $55.00,130.00$ \\
\hline & \multirow[t]{3}{*}{ POD-4 } & Mean (SD) & $79.77(12.22)$ & 78.01 (12.32) & $78.91(12.26)$ \\
\hline & & Median (Q1, Q3) & $78.00(72.00,88.00)$ & $78.00(70.00,86.00)$ & $78.00(71.00,86.00)$ \\
\hline & & Min, Max & $53.00,112.00$ & $51.00,110.00$ & $51.00,112.00$ \\
\hline \multirow{15}{*}{$\begin{array}{l}\text { Systolic } \\
\text { pressure } \\
(\mathrm{mmHg})\end{array}$} & \multirow[t]{3}{*}{ Pre-operation } & Mean (SD) & $128.23(16.54)$ & $126.52(15.64)$ & 127.39 (16.08) \\
\hline & & Median (Q1, Q3) & $125.00(117.00,141.00)$ & $125.50(116.00,135.00)$ & $125.00(117.00,139.00)$ \\
\hline & & Min, Max & $98.00,172.00$ & $95.00,182.00$ & $95.00,182.00$ \\
\hline & \multirow[t]{3}{*}{ POD-1 } & Mean (SD) & $122.73(17.60)$ & $123.97(20.79)$ & $123.36(19.22)$ \\
\hline & & Median (Q1, Q3) & $120.00(109.00,136.00)$ & $121.00(108.00,140.00)$ & $121.00(109.00,138.00)$ \\
\hline & & Min, Max & $90.00,700.00$ & $88.00,186.00$ & $88.00,186.00$ \\
\hline & \multirow[t]{3}{*}{ POD-2 } & Mean (SD) & $125.03(17.00)$ & $127.42(15.74)$ & $126.19(16.38)$ \\
\hline & & Median (Q1, Q3) & $125.00(114.00,135.00)$ & $129.00(118.00,136.00)$ & $127.00(116.00,135.00)$ \\
\hline & & Min, Max & $94.00,180.00$ & $91.00,164.00$ & $91.00,180.00$ \\
\hline & POD-3 & Mean (SD) & $127.35(16.02)$ & $124.27(16.32)$ & $125.66(16.17)$ \\
\hline & & Median (Q1, Q3) & $127.00(117.00,138.00)$ & $121.50(113.00,137.00)$ & $123.00(114.00,137.00)$ \\
\hline & & Min, Max & $99.00,159.00$ & $93.00,162.00$ & $93.00,162.00$ \\
\hline & POD-4 & Mean (SD) & $131.06(20.89)$ & $129.97(17.59)$ & $130.52(19.20)$ \\
\hline & & Median (Q1, Q3) & $127.00(119.00,147.00)$ & $130.00(116.50,142.50)$ & $129.00(117.00,143.00)$ \\
\hline & & Min, Max & $88.00,178.00$ & $102.00,167.00$ & $88.00,178.00$ \\
\hline Diastolic & Pre-operation & Mean (SD) & $79.78(10.03)$ & $77.81(9.70)$ & $78.81(9.89)$ \\
\hline $\begin{array}{l}\text { pressure } \\
(\mathrm{mmHa})\end{array}$ & & Median (Q1, Q3) & $78.00(73.00,86.00)$ & $78.00(71.00,85.00)$ & $78.00(72.00,85.00)$ \\
\hline & & Min, Max & $57.00,105.00$ & $60.00,106.00$ & $57.00,106.00$ \\
\hline & POD-1 & Mean (SD) & $72.88(10.96)$ & $73.88(12.78)$ & $73.38(11.88)$ \\
\hline & & Median (Q1, Q3) & $73.00(65.00,81.00)$ & $73.00(64.00,83.00)$ & $73.00(65.00,82.00)$ \\
\hline & & Min, Max & $52.00,97.00$ & $41.00,113.00$ & $41.00,113.00$ \\
\hline & POD-2 & Mean (SD) & $76.33(9.57)$ & $77.53(14.97)$ & $76.92(12.46)$ \\
\hline & & Median (Q1, Q3) & $77.00(71.00,81.00)$ & $76.50(71.00,80.00)$ & $77.00(71.00,80.00)$ \\
\hline & & Min, Max & $59.00,108.00$ & $53.00,168.00$ & $53.00,168.00$ \\
\hline & POD-3 & Mean (SD) & $79.60(9.81)$ & $76.25(9.95)$ & $77.79(9.98)$ \\
\hline & & Median (Q1, Q3) & $79.00(70.00,87.00)$ & $74.00(69.00,86.00)$ & $78.00(70.00,86.00)$ \\
\hline & & Min, Max & $60.00,97.00$ & $59.00,99.00$ & $59.00,99.00$ \\
\hline & POD-4 & Mean (SD) & $79.55(10.67)$ & $78.25(9.31)$ & $78.91(9.97)$ \\
\hline & & Median (Q1, Q3) & $78.00(70.00,88.00)$ & $78.50(71.00,85.00)$ & $78.00(70.00,86.00)$ \\
\hline & & Min, Max & $58.00,99.00$ & $62.00,95.00$ & $58.00,99.00$ \\
\hline
\end{tabular}


Table S2 Analysis of Serological examinations (SS population)

\begin{tabular}{|c|c|c|c|c|c|}
\hline Index & Classification & Statistics & Group intervention & Group control & Total \\
\hline \multirow[t]{12}{*}{ WBC (10^9/L) } & Pre-operation & Mean (SD) & $6.46(1.87)$ & $6.42(2.20)$ & $6.44(2.04)$ \\
\hline & & $\begin{array}{l}\text { Median } \\
(\mathrm{Q} 1, \mathrm{Q} 3)\end{array}$ & $6.22(4.94,7.64)$ & $5.94(4.94,7.24)$ & $6.11(4.94,7.51)$ \\
\hline & & Min, Max & $3.37,12.02$ & $3.41,18.22$ & $3.37,18.22$ \\
\hline & POD-1 & Mean (SD) & $10.67(3.56)$ & $10.02(2.91)$ & $10.35(3.26)$ \\
\hline & & $\begin{array}{c}\text { Median (Q1, } \\
\text { Q3) }\end{array}$ & $10.05(7.98,12.61)$ & $9.57(7.50,11.91)$ & $9.82(7.91,12.14)$ \\
\hline & & Min, Max & $4.60,21.33$ & $6.16,18.45$ & $4.60,21.33$ \\
\hline & POD-3 & Mean (SD) & $8.25(2.68)$ & $7.79(2.60)$ & $8.02(2.65)$ \\
\hline & & $\begin{array}{c}\text { Median (Q1, } \\
\text { Q3) }\end{array}$ & $8.09(5.95,9.93)$ & $7.65(5.82,9.53)$ & $7.87(5.89,9.85)$ \\
\hline & & Min, Max & $3.79,14.89$ & $1.72,16.96$ & $1.72,16.96$ \\
\hline & POD-5 & Mean (SD) & $7.35(3.06)$ & $6.97(2.46)$ & $7.16(2.77)$ \\
\hline & & $\begin{array}{c}\text { Median (Q1, } \\
\text { Q3) }\end{array}$ & $6.68(5.12,8.87)$ & $6.74(5.44,7.85)$ & $6.70(5.35,8.12)$ \\
\hline & & Min, Max & $3.00,18.74$ & $2.44,15.50$ & $2.44,18.74$ \\
\hline \multirow[t]{13}{*}{ HGB (g/L) } & Pre-operation & Mean (SD) & $119.58(25.00)$ & $131.14(119.17)$ & $125.36(86.05)$ \\
\hline & & $\begin{array}{c}\text { Median (Q1, } \\
\text { Q3) }\end{array}$ & $123.00(109.00,137.00)$ & $119.50(104.00,134.00)$ & $121.00(106.00,135.00)$ \\
\hline & & Min, Max & $10.00,165.00$ & $68.00,1230.00$ & $10.00,1230.00$ \\
\hline & POD-1 & Mean (SD) & $112.66(18.81)$ & $111.72(20.06)$ & 112.19 (19.39) \\
\hline & & $\begin{array}{c}\text { Median (Q1, } \\
\text { Q3) }\end{array}$ & $116.00(101.00,129.00)$ & $113.00(100.00,127.00)$ & $114.00(100.00,127.00)$ \\
\hline & & Min, Max & $70.00,149.00$ & $69.00,159.00$ & $69.00,159.00$ \\
\hline & POD-3 & Mean (SD) & $108.05(18.04)$ & $104.84(19.95)$ & $106.43(19.03)$ \\
\hline & & $\begin{array}{c}\text { Median (Q1, } \\
\text { Q3) }\end{array}$ & $108.50(97.00,118.50)$ & $107.00(91.00,117.00)$ & $108.00(93.00,118.00)$ \\
\hline & & Min, Max & $66.00,148.00$ & $31.00,153.00$ & $31.00,153.00$ \\
\hline & POD-5 & Mean (SD) & $108.25(18.14)$ & $106.98(18.79)$ & $107.62(18.39)$ \\
\hline & & $\begin{array}{c}\text { Median (Q1, } \\
\text { Q3) }\end{array}$ & $109.00(94.50,120.50)$ & $106.50(95.50,121.00)$ & $108.00(95.00,121.00)$ \\
\hline & & Min, Max & $69.00,160.00$ & $66.00,147.00$ & $66.00,160.00$ \\
\hline & & $\mathrm{N}$ (n miss) & $90(1)$ & $89(1)$ & $179(2)$ \\
\hline \multirow[t]{12}{*}{ PLT (10^9/L) } & Pre-operation & Mean (SD) & 280.23 (119.69) & $262.24(90.86)$ & 271.28 (106.42) \\
\hline & & $\begin{array}{c}\text { Median (Q1, } \\
\text { Q3) }\end{array}$ & $252.00(202.00,328.00)$ & $254.00(205.00,305.00)$ & $254.00(202.00,315.00)$ \\
\hline & & Min, Max & $25.00,754.00$ & $59.00,517.00$ & $25.00,754.00$ \\
\hline & POD-1 & Mean (SD) & $247.20(88.10)$ & 232.08 (81.62) & $239.69(85.04)$ \\
\hline & & $\begin{array}{c}\text { Median (Q1, } \\
\text { Q3) }\end{array}$ & $231.50(180.00,302.00)$ & $227.00(176.00,287.00)$ & $229.00(180.00,288.00)$ \\
\hline & & Min, Max & $93.00,606.00$ & $60.00,425.00$ & $60.00,606.00$ \\
\hline & POD-3 & Mean (SD) & $226.94(90.18)$ & 213.11 (74.89) & 220.02 (82.93) \\
\hline & & $\begin{array}{c}\text { Median (Q1, } \\
\text { Q3) }\end{array}$ & $210.00(170.00,268.00)$ & $213.00(162.00,255.00)$ & $210.00(166.00,260.00)$ \\
\hline & & Min, Max & $93.00,652.00$ & $57.00,446.00$ & $57.00,652.00$ \\
\hline & POD-5 & Mean (SD) & $239.17(85.97)$ & $231.37(79.00)$ & $235.31(82.28)$ \\
\hline & & $\begin{array}{c}\text { Median (Q1, } \\
\text { Q3) }\end{array}$ & $226.00(182.50,286.50)$ & $236.00(171.00,258.00)$ & $230.00(177.00,280.00)$ \\
\hline & & Min, Max & $85.00,569.00$ & $72.00,445.00$ & $72.00,569.00$ \\
\hline
\end{tabular}

POD-1, postoperative day 1st; POD-3, postoperative day 3rd; POD-5, postoperative day 5th; WBC, white blood cell; HGB, Hemoglobin; PLT, Platelet. 